\title{
Stochastic transitivity and cancellation of preferences between bitter-sweet solutions'
}

\author{
Don H. MeLaughlin 2 and R. Dunean Luce \\ UNIVERSITY OF PENNSYLVANIA
}

\begin{abstract}
Preferences between 25 pairs of stimuli composed from 25 different sucrose-quinine sulphate solutions were obtained from three Ss in an attempt to test the product rule; strong, moderate, and weak stochastic transitivity; and strong, moderate, and weak stochastic cancellation. The data seem to support strong, and hence moderate and weak, stochastic transitivity as well as moderate, and hence, weak stochastic cancellation. The product rule and strong stochastic cancellation appear to be more dubious.
\end{abstract}

\section{Problem}

The mathematical literature on preference includes several proposals for restrictions among the binary preference probabilities $p(x, y), p(y, z)$, and $p(x, z)$, where $x, y$, and $z$ are stimuli and $p(x, y)$ denotes the probability that $\mathrm{x}$ is judged preferable to $\mathrm{y}$, etc. For a summary, see Luce \& Suppes (1965, pp. 339-346). The strongest constraint is the product rule:

$$
\begin{aligned}
p(x, z)= & p(x, y) p(y, z) /[p(x, y) p(y, z) \\
& +p(z, y) p(y, x)] .
\end{aligned}
$$

It implies three successively weaker probabilistic generalizations of transitivity known as strong, moderate, and weak stochastic transitivity: if $\mathrm{p}(\mathrm{x}, \mathrm{y}) \geq 1 / 2$ and $\mathrm{p}(\mathrm{y}, \mathrm{z}) \geq 1 / 2$, then

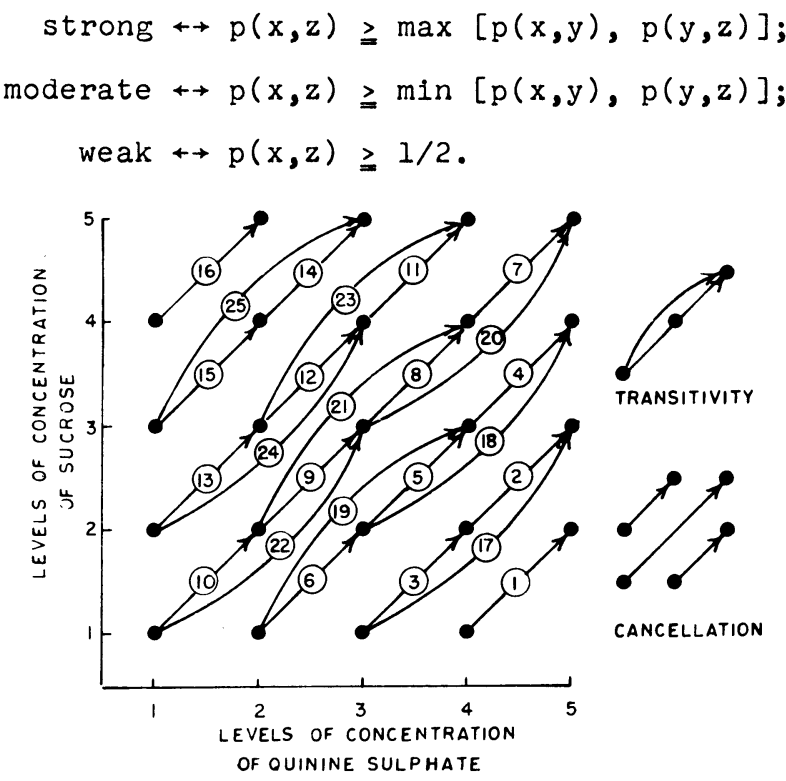

Fig. 1. On the left are shown the 25 test comparisons among stimuli composed of one of five levels of sucrose and one of five levels of quinine sulphate. On the right are shown typical test configurations for transitivity and for cancellation. The arrows point in the direction of greater concentration.
The abbreviations SST, MST, and WST are used.

When the stimuli consist of two (or more) dimensions that can be varied independently, e. g., in this study, solutions containing both sucrose and quinine sulphate, then additional relations are conceivable. Perhaps the most important algebraic one is cancellation, which must be met if preferences can be represented by a numerical utility function that is additive over the dimensions; moreover, together with transitivity of preference it is nearly sufficient to imply such a numerical representation provided that each dimension is sufficiently finely graded (see Luce \& Suppes, pp. 267-272). It also has three probabilistic generalizations known as strong, moderate, and weak stochastic concellation (SSC, MSC, WSC): if $p[(a, x),(f, s)] \geqq 1 / 2$ and $p[(f, r)$, $(b, x)] \geqq 1 / 2$, then

$$
\begin{aligned}
& \text { strong } \leftrightarrow p[(a, r),(b, s)] \geq \\
& \quad \max \{p[(a, x),(f, s)], p[(f, r),(b, x)]\} ; \\
& \operatorname{moderate} \leftrightarrow p[(a, r),(b, s)] \geq \\
& \quad \min \{p[(a, x),(f, s)], p[(f, r),(b, x)]\} ;
\end{aligned}
$$

weak $\rightarrow p[(a, r),(b, s)] \geqq 1 / 2$,

where $f$ and $x$ are the "cancelled" elements.

This experiment was designed to see which, if any, of these restrictions are applicable to taste preferences of two dimensional, bitter-sweet stimuli.

\section{Method}

Design. The stimulus solutions contained one of five amounts of quinine sulphate, $0.025,0.037,0.055,0.083$, or $0.125 \mathrm{mg}$, plus one of five amounts of sucrose, $0.031,0.045,0.064,0.091$, or $0.125 \mathrm{gm}$, per $\mathrm{gm}$ of solution. Labeling these levels 1 through 5 in increasing concentration, the 25 test pairs are shown by numbered arrows in Fig. 1. Typical sets of three comparisons to test transitivity and cancellation are shown at the right; there are 9 of each. Note that all comparisons are of a lower vs. a greater concentration on both components. Were choices based on this psychophysical judgment, both WST and WSC would follow automatically, and if probability increased with the degree of separation, then both SST and SSC would hold. Since pilot work suggested this might be a problem, we attempted to break this set by presenting 25 non-test comparisons made up of the relatively sweet vs. relatively bitter pairs obtained by rotating the grid of arrows shown in Fig. 1 through $90^{\circ}$ about the central point $(3,3)$.

In each session, which lasted between 55 and 75 min., each test pair was presented three times and each of the non-test pairs, once. There were 17 sessions. The order of presentation was randomized subject to the condition that 25 of the 51 test presentations occurred in one order.

Subjects. Three college undergraduates were paid to participate. Each had participated previously in psychological experiments, but neither in a taste nor in a preference study.

Procedure. At least $30 \mathrm{~min}$. prior to a session, $3 \mathrm{gm}$ amounts of the 25 refrigerated solutions were placed in $1 \mathrm{oz}$ paper cups and maintained at room temperature. Each trial consisted of six 5-sec. 


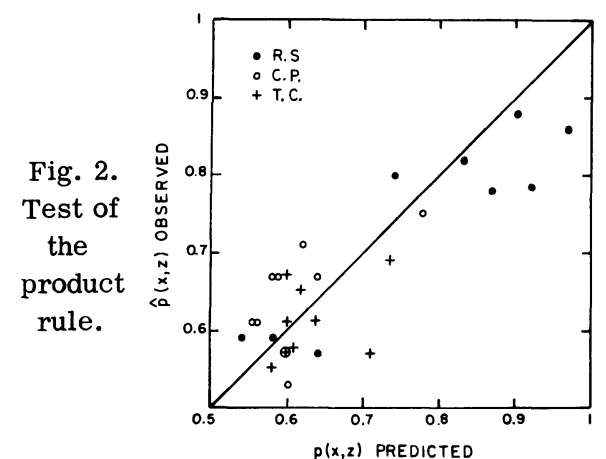

intervals which were timed by glow-bulb-capacitor timer. Each trial began when $\mathrm{S}$ was ready. Each $\mathrm{S}$ worked out a rhythmic scheme for his activities and maintained a consistent pace in the following order: (1) water was held in the mouth and expectorated at end of period; (2) solution from the left of two cups was placed on the front of tongue; (3) solution was expectorated and S waited; (4) water was held in mouth and expectorated at end of period; (5) solution from the right cup was placed on the front of tongue; and (6) solution was expectorated and $\mathrm{S}$ wrote which solution, first or second, was preferred.

\section{Results}

Using the numbering of Fig. 1, the estimated preference probabilities are shown in Table 1 . Of the 16 adjacent pairs, 10,8 , and 5 , and of the 9 non-adjacent pairs, 7,5 , and 3 , respectively, favored the more concentrated stimulus, which suggests that the Ss did not base their choices entirely on concentration.

Figures 2-5 compare the estimates $\hat{\mathrm{p}}(\mathrm{x}, \mathrm{z})$ with those predicted from the other relevant estimates by, respectively, the product rule, SST, SSC, and MSC. The dotted line in Fig. 3 represents one standard deviation below the $45^{\circ}$ line on the assumption of only binomial variability in $\mathrm{p}(\mathrm{x}, \mathrm{z})$.

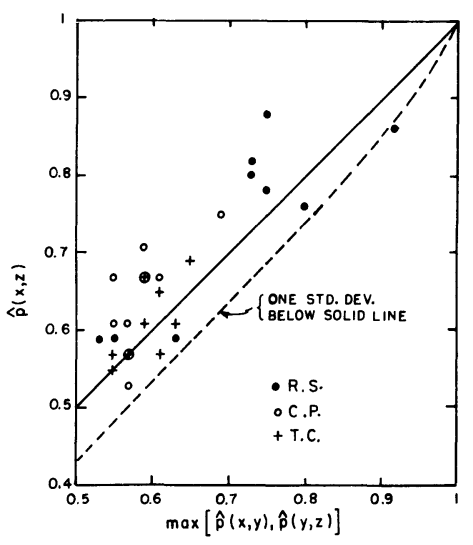

Fig. 3i. Test of strong stochastic transitivity.

\section{Diseussion}

The variability in Fig. 2 and the over prediction of $p(x, z)$ for values in excess of 0.75 makes suspect the product rule. It should be noted, however, that five of the six points above 0.75 are due to one S. Figure 3 provides adequate support for SST, and, of course, stronger support for MST and WST. Figure 4 casts considerable doubt upon SSC, but Fig. 5 lends support to MSC and almost complete support for WSC. The
Table 1. Estimated Preference Probabilities for the Stronger Solution in the 25 Test Pairs. The numbering of the pairs is shown in Fig. 1. Decimal points are omitted.

\begin{tabular}{|c|c|c|c|c|c|c|c|c|c|c|c|c|c|}
\hline \multicolumn{14}{|c|}{ Pair } \\
\hline Subject & 1 & 2 & 3 & 4 & 5 & 6 & 7 & 8 & 9 & 10 & 11 & 12 & 13 \\
\hline R. S. & 14 & 35 & 27 & 45 & 47 & 59 & 57 & 49 & 80 & 75 & 69 & 75 & 75 \\
\hline C. P. & 31 & 45 & 47 & 41 & 47 & 71 & 33 & 53 & 57 & 57 & 49 & 61 & 69 \\
\hline T. C. & 31 & 41 & 35 & 49 & 41 & 49 & 51 & 39 & 39 & 65 & 45 & 55 & 55 \\
\hline \multicolumn{14}{|c|}{ Pair } \\
\hline Subject & & 14 & 15 & 16 & 17 & 18 & 19 & 20 & 21 & 22 & 23 & 24 & 25 \\
\hline R. S. & & 73 & 92 & 76 & 18 & 41 & 51 & 63 & 73 & 76 & 78 & 88 & 86 \\
\hline C. P. & & 39 & 57 & 53 & 33 & 33 & 59 & 39 & 53 & 57 & 55 & 75 & 49 \\
\hline T. C. & & 47 & 57 & 47 & 31 & 39 & 33 & 37 & 43 & 51 & 47 & 57 & 57 \\
\hline
\end{tabular}

failure of SSC reinforces the earlier argument that Ss did not simply judge the comparative concentrations.

At least three possible artifacts may vitiate these conclusions. Response bias: there was some tendency to avoid the first (left) stimulus; the choices were 40,36 , and 44 percent, respectively. This fact necessarily increased the variability slightly beyond binomial. Order of presentation: if the probabilities are estimated separately when the stronger solution was presented first and when it was second, the former estimates are smaller in 24 cases for Ss R. S. and C. P. and in 19 cases for T. C. out of the 25 possible. Again this must have increased the variability. In addition it suggests that more detailed analyses are needed to obtain a fuller understanding of preference judgments. Learning: separating the data from the first and second halves of the experiment did not reveal any consistent trends. It is not clear whether the increases in variability are sufficient to render the product rule and SSC acceptable.

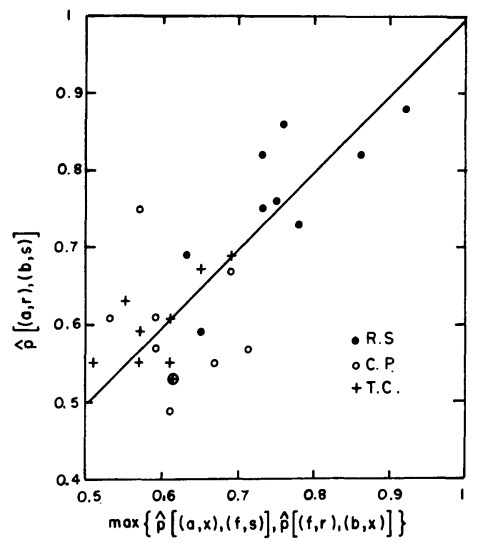

Fig. 4. Test of strong stochastic cancellation.

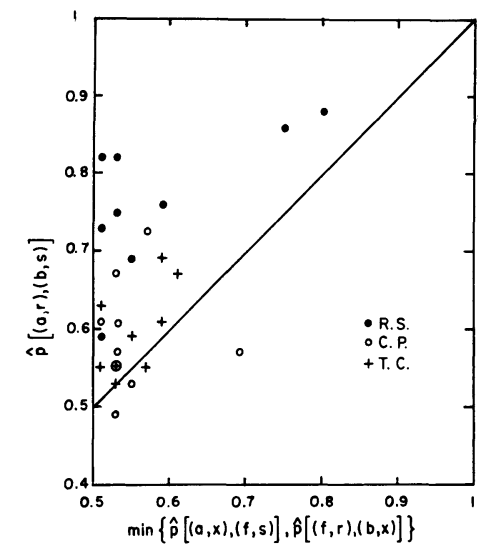

Fig. 5. Test of moderate stochastic cancellation.

Referenee

LUCE, R. D., \& SUPPES, P. Preference, utility, and subjective probability. In R. D. Luce, R. R. Bush, \& E. Galanter (Eds.), Handbook of mathematical psychology, Vol. III. New York: Wiley, 1965. Pp. 249-410.

\section{Notes}

1. This research was supported in part by the National Science Foundation Grant NSF GB 1462 to the University of Pennsylvania. Our thanks to F. W. Irwin for his comments.

2. Now a graduate student in the Department of Psychology, Carnegie Institute of Technology. 